\title{
Radiolabeled Amino Acids for Oncologic Imaging
}

\author{
Chaofeng Huang and Jonathan McConathy \\ Department of Radiology, Washington University School of Medicine, St. Louis, Missouri
}

Radiolabeled amino acids (AAs) are an important class of imaging agents for PET and SPECT that target the increased levels of $A A$ transport by many tumor cells. System $L A A$ transport has been a major focus of tracer development, and work in this field has led to several tracers that are effective for imaging brain tumors. Emerging data also support the use of certain radiolabeled AAs for neuroendocrine tumors and prostate cancer. Recently, new ${ }^{18}$ F-labeled AAs have been developed that target transporters other than system L, including system A, glutamine, $x C T$, and cationic AA transporters. This review provides an overview of this class of molecular imaging agents and highlights the current status of oncologic imaging with radiolabeled AAs in terms of tracer considerations and key clinical applications.

Key Words: PET; SPECT; amino acids; tumor imaging

J Nucl Med 2013; 54:1007-1010

DOI: 10.2967/jnumed.112.113100

Radiolabeled amino acids (AAs) have been used for human imaging studies for decades, and there is active investigation in this field in part due to recent insights into the role of AA transporters and AAs in tumor metabolism. Beginning in the late 1990s, the genes coding for individual AA transporters have been cloned and characterized. There is now abundant evidence that certain transporter proteins are upregulated in a wide range of neoplastic cells, confer prognostic information, and are potential targets of therapy. The importance of certain AAs, such as leucine, glutamine, and arginine in neoplastic cells, has guided the development of new radiolabeled AAs to probe cancer metabolism.

\section{GENERAL CONSIDERATIONS}

Natural $\alpha$-AAs are an important class of small molecules that play vital roles in many cellular processes including protein synthesis, energy metabolism, cell signaling, carbon sources for cell growth, and neurotransmission. In oncologic imaging applications, radiolabeled AAs target the increased levels of AA transport that occur in many tumor cells compared with normal tissues, thought to be mediated by increased cell surface levels of AA transporters. Although some radiolabeled AAs are incorporated into proteins or have other metabolic fates, tumor uptake and imaging properties

Received Jan. 15, 2013; revision accepted May 2, 2013.

For correspondence or reprints contact: Jonathan McConathy, Washington University School of Medicine, Department of Radiology, 510 S. Kingshighway

Blvd., Campus Box 8223, St. Louis, MO 63110.

E-mail: mcconathyj@mir.wustl.edu

Published online May 24, 2013.

COPYRIGHT (C) 2013 by the Society of Nuclear Medicine and Molecular Imaging, Inc. reflect primarily the rate and mechanism of transport of the AA in most instances.

AA transporters are membrane-associated proteins coded by family members of the solute carrier (SLC) series of genes that mediate the transfer of AAs across cell membranes. AA transporter families have traditionally been characterized functionally as transport systems (e.g., system L, system A, system N, system ASC, and system xCT), many of which contain multiple family members. Over 20 distinct AA transport systems have been identified, with different substrate specificities, tissue expression patterns, dependence on cotransport of ions such as sodium, hormonal regulation, mechanisms of transport, and biologic significance in cancer. Two AA transporters, the system L transporter type 1 (LAT1, SLC7A5) and the system ASC transporter type 2 (ASCT2, SLC1A5), are upregulated in a wide range of human cancers, are associated with worse prognosis, and are potential targets for therapy $(1,2)$. There is growing evidence that these and other AA transporters and their substrates interact with the mammalian target of rapamycin pathway, which regulates protein synthesis and cell proliferation $(3,4)$. Radiolabeled AAs could potentially be used to monitor response to therapies targeting mammalian target of rapamycin signaling.

Structural and radionuclide considerations often influence the design of novel radiolabeled AAs and the selection of specific AA tracers for a study. Natural AAs or their close analogs may be preferred if specific aspects of their transport or biochemistry are important for a particular application. In many instances, nonnatural, nonmetabolized AAs such as $O-\left(2-{ }^{18} \mathrm{~F}\right.$-fluoroethyl)-Ltyrosine (FET) and anti-1-amino-3-18 F-fluorocyclobutane-1-carboxylic acid (FACBC) have advantages over natural AAs, including the ability to incorporate longer-lived radionuclides such as ${ }^{18} \mathrm{~F}$ and ${ }^{123} \mathrm{I}$, simplified radiosynthetic methods, and lack of radiolabeled metabolite formation, which simplifies kinetic analysis and avoids potentially confounding accumulation of activity in nontarget tissues. All AAs theoretically can be labeled with ${ }^{11} \mathrm{C}$, and the 20 min half-life of ${ }^{11} \mathrm{C}$ is well suited to the time to peak tumor uptake, which typically occurs within 15-20 min after administration. The use of ${ }^{18} \mathrm{~F}$ takes advantage of the longer 110 -min half-life of this radionuclide, which makes batch production and remote distribution feasible. However, ${ }^{18} \mathrm{~F}$ is not present in natural mammalian AAs and in some cases substantially alters the biologic properties of an AA. ${ }^{123}$ I (half-life, $13.2 \mathrm{~h}$ ) has been used less frequently than ${ }^{11} \mathrm{C}$ or ${ }^{18} \mathrm{~F}$ for labeling AAs, but several ${ }^{123}$ I-labeled AAs have been developed for SPECT imaging, including 3-123I-iodo- $\alpha$-methyl tyrosine (IMT).

\section{APPLICATIONS OF RADIOLABELED AAS FOR ONCOLOGIC IMAGING}

A wide range of radiolabeled AAs has been developed and used for preclinical and clinical oncologic imaging studies (5). The 
structures of selected radiolabeled AAs for oncologic imaging are depicted in Figure 1. Much of this work has focused on aromatic AA derivatives, including ${ }^{18} \mathrm{~F}$-FET, $6{ }^{18} \mathrm{~F}$-fluoro-3,4-dihydoxy-Lphenylalanine (FDOPA), ${ }^{123} \mathrm{I}$-IMT, and $3-{ }^{18} \mathrm{~F}$-fluoro- $\alpha$-methyltyrosine (FMT), which target system L AA transport. Other nonaromatic system $\mathrm{L}$ substrates include ${ }^{11} \mathrm{C}$-MET and the cycloleucine analog, ${ }^{18} \mathrm{~F}-\mathrm{FACBC}$. Tracers targeting other AA transport systems, including system A, glutamine, glutamate, and cationic AA transporters, have been developed but have not been as extensively evaluated in human studies.

\section{Brain Tumors}

Neuroimaging plays a critical role in primary and metastatic brain tumors, including diagnosis, treatment planning, monitoring response to therapy, and assessing for recurrence. Contrast-enhanced MR imaging is the primary imaging modality for evaluating brain tumors but has limited accuracy for defining tumor boundaries and volumes, evaluating the nonenhancing portions of gliomas, and differentiating treatment effects such as radiation necrosis from viable tumor. ${ }^{18} \mathrm{~F}$-FDG PET is limited by high physiologic uptake in normal brain, and both MR imaging and ${ }^{18} \mathrm{~F}-\mathrm{FDG}$ PET have limited specificity for distinguishing recurrent tumor from radiation necrosis. Two major advantages of system L AA transport substrates for brain tumor imaging are their ability to cross the intact $\mathrm{BBB}$ and visualize the entire tumor volume and their relatively low uptake and retention in normal brain, compared with ${ }^{18} \mathrm{~F}$-FDG. These properties of radiolabeled AAs targeting system $\mathrm{L}$ transport complement contrast-enhanced MR imaging by providing a more accurate visualization of the entire tumor volume.
Of the radiolabeled AAs targeting system $\mathrm{L},{ }^{11} \mathrm{C}-\mathrm{MET}$ and ${ }^{18} \mathrm{~F}$ FET have the most data supporting their use for imaging brain gliomas $(6,7)$. An example of ${ }^{11} \mathrm{C}$-MET uptake in recurrent glioblastoma is shown in Figure 2. Other tracers used for this application include ${ }^{123} \mathrm{I}$-IMT and ${ }^{18} \mathrm{~F}$-FDOPA $(8)$. There are relatively few studies directly comparing these tracers for tumor imaging, but the available data suggest they behave similarly in terms of tumor visualization. Both ${ }^{18} \mathrm{~F}$-FET and ${ }^{123} \mathrm{I}$-IMT are metabolically stable, which may be advantageous for the analysis of tracer kinetics. There is a substantial body of data showing that ${ }^{11} \mathrm{C}-\mathrm{MET}$ and ${ }^{18}$ F-FET PET studies can improve the diagnostic yield of brain tumor biopsy, more accurately define gross tumor volumes and margins than MR imaging alone, and follow response to chemotherapy in nonenhancing gliomas $(6,9)$. Changes in tumor volumes are typically observed earlier with AA PET than with MR imaging, particularly in nonenhancing gliomas, and appear to be more predictive of response to therapy than changes in absolute tracer uptake. There are also studies suggesting that the kinetics of ${ }^{18} \mathrm{~F}-\mathrm{FET}$ uptake in gliomas can distinguish high-grade from low-grade tumors (10). ${ }^{18} \mathrm{~F}$-FET and ${ }^{11} \mathrm{C}$-MET may also be useful for distinguishing recurrent brain tumors from radiation necrosis after radiation therapy $(11,12)$, but additional data are needed to better define the accuracy of these tracers for this purpose in clinical practice.

\section{Neuroendocrine Tumors}

Neuroendocrine tumors encompass a range of neoplasms, including carcinoid tumors, pancreatic islet cell tumors, pheochromocytomas and paragangliomas, neuroblastoma, and small cell lung cancer. A common feature of these tumors is the potential for the production of biologically active substances that can lead to severe symptoms, morbidity, and even death. The accurate localization of the primary tumor and metastases is important for selecting the appropriate therapeutic approach in these patients. Uptake of ${ }^{18} \mathrm{~F}$ FDG in neuroendocrine tumors is variable, and well-differentiated neuroendocrine tumors often have low levels of ${ }^{18} \mathrm{~F}-\mathrm{FDG}$ uptake. Of the radiolabeled AAs, ${ }^{18} \mathrm{~F}$-FDOPA has been used the most extensively in neuroendocrine tumors. The enzyme aromatic AA decarboxylase (AADC) is active in many neuroendocrine tumors, and metabolism of ${ }^{18} \mathrm{~F}$-FDOPA by AADC likely contributes to its favorable imaging properties because other system L substrates are not as effective for neuroendocrine tumor imaging (13).

Of the neuroendocrine tumors, ${ }^{18} \mathrm{~F}$ FDOPA appears to best suited for imaging carcinoid tumors, pancreatic insulinomas, and neuroblastoma and can detect lesions not identified by CT, MR imaging, or other tracers used clinically for neuroendocrine tumor imaging $(13,14)$. Studies comparing the utility of FDOPA with that of somatostatin receptor scintigraphy have demonstrated that somatostatin receptor scintigraphy typically demonstrates higher sensitivity for neuroendocrine tumor detection on a per-lesion basis, although ${ }^{18} \mathrm{~F}$ - 


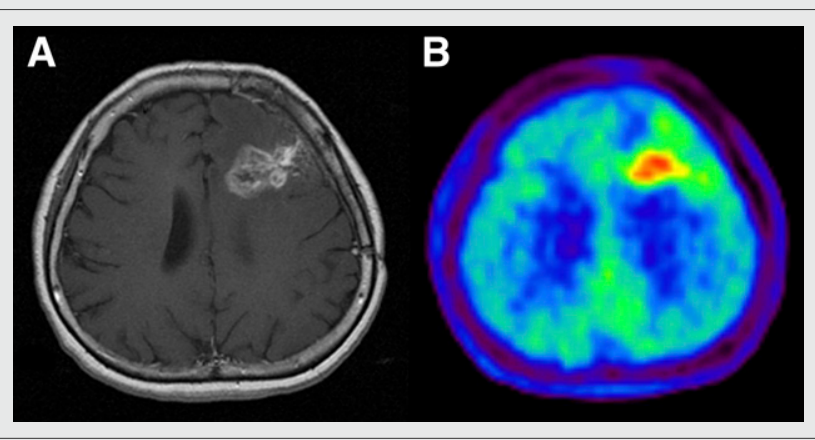

FIGURE 2. Glioblastoma imaging with ${ }^{11} \mathrm{C}-\mathrm{MET}$. Contrastenhanced T1-weighted MR (A) and ${ }^{11} \mathrm{C}$-MET PET (B) images in patient with glioblastoma treated with surgical resection and radiation therapy, now with suspected recurrence. ${ }^{11} \mathrm{C}-\mathrm{MET}$ PET image demonstrates focally increased uptake within area of contrast enhancement on MR, and this lesion was pathologically proven to be recurrent glioblastoma on resection. In patients with radiation necrosis, lower ${ }^{11} \mathrm{C}-\mathrm{MET}$ uptake was observed (images not shown). (Reprinted from (12).)

FDOPA can detect some neuroendocrine tumors that are not detected with somatostatin receptor scintigraphy $(15,16)$.

\section{Prostate Cancer}

Prostate cancer is a common malignancy in men and the second leading cause of cancer-related mortality in the United States. The management of prostate cancer is challenging, because many prostate cancers are indolent whereas others are aggressive. Conventional imaging with CT and MR imaging has limited accuracy for the detection of the primary tumor and locoregional lymph node metastases. Unlike many other cancers, ${ }^{18}$ F-FDG uptake in prostate cancer is often low. The clinically available monoclonal antibody ${ }^{111}$ In-capromab, targeting the prostate membrane-specific antigen,

is used for the detection of recurrent prostate cancer but has limited accuracy. In response to the great need for improved imaging techniques, several classes of PET tracers have been developed for prostate cancer imaging. Recent studies with anti- ${ }^{18}$ F-FACBC have shown that this AA tracer can accurately detect prostate cancer within the gland itself and in pelvic lymph node metastases with specificity and sensitivity superior to ${ }^{111} \mathrm{In}$-capromab SPECT/ CT (17). An example of ${ }^{18}$ F-FACBC PET/CT in a patient with recurrent prostate cancer is shown in Figure 3. One advantage of anti-3- ${ }^{18}$ F-FACBC over other radiolabeled AAs is that the relatively low levels of urinary excretion simplify imaging of the pelvis. In vitro uptake studies on prostate cancer cell lines indicate that ${ }^{18} \mathrm{~F}$-FACBC is a substrate for both LAT1 and ASCT2 (18).

\section{Other Cancers}

In general, radiolabeled AAs have not performed as well as ${ }^{18} \mathrm{~F}$ FDG PET for detection and staging of cancers other than those of the brain, neuroendocrine system, and prostate. The main limitation is decreased sensitivity for the detection of primary tumors and metastases, compared with ${ }^{18}$ F-FDG. One of the potential limitations of system L substrates is the ability of these transporters to mediate both influx and efflux of substrates, limiting the tumor-tobackground ratios that can be achieved. There is growing interest in using radiolabeled AAs to better characterize the biologic behavior of cancers. These types of applications have the potential to provide information relevant to diagnosis, prognosis, and therapy. For example, the amount of uptake of the system L substrate $3-{ }^{18}$ F-FMT in lung adenocarcinoma is negatively correlated with survival (19). These results fit with the observation in many human cancers that higher levels of LAT1 messenger RNA and protein are associated with higher mortality.

\section{EMERGING CLASSES OF RADIOLABELED AAS}

The roles of radiolabeled AAs that are substrates for transporters other than system $\mathrm{L}$ are less well

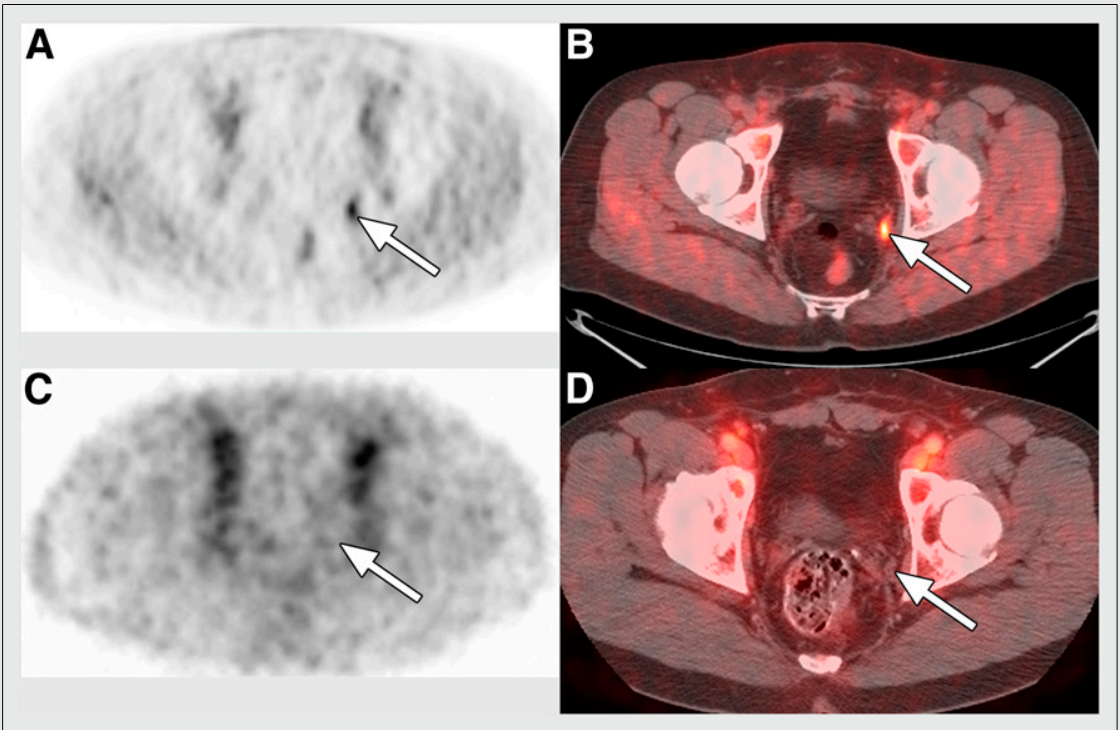

FIGURE 3. Prostate cancer imaging with ${ }^{18} \mathrm{~F}$-FACBC. ${ }^{18} \mathrm{~F}$-FACBC PET (A) and ${ }^{18} \mathrm{~F}-\mathrm{FACBC}$ $\mathrm{PET} / \mathrm{CT}(\mathrm{B})$ images from a patient with suspected recurrence of prostate cancer demonstrate focal uptake in left pelvic lymph node metastasis that measures less than $1 \mathrm{~cm}$ in short-axis diameter (indicated with arrows). Biopsy demonstrated recurrent prostate cancer in this location. In contrast, ${ }^{111}$ In-capromab pendetide SPECT (C) and SPECT/CT (D) images do not show uptake in this metastatic lymph node (false-negative). (Reprinted with permission from (17).) defined. Recently, AA tracer development has explored several different transport systems for oncologic imaging, including system A (20), system xCT $(21,22)$, glutamine transporters $(23,24)$, and cationic AA transporters $(25,26)$. The availability of these new tracers will provide important tools to probe the roles of these AA transport systems in specific cancers and for particular applications.

Each of these transport systems has unique biologic features and the potential to provide clinically useful information through PET and SPECT imaging studies. System A transport is upregulated during cell growth and by a variety of cell stressors. Additionally, the fact that system A can concentrate its substrates intracellularly may provide higher tumor uptake than can be achieved with system L substrates. Glutamine is a key metabolic substrate that can provide energy, tricarboxylic acid cycle intermediates, and carbon for cell growth (3). As mentioned previously, the ASCT2 transporter plays important roles in tumor biology and is thought to 
be a key mediator of glutamine uptake by many tumor cells. System $\mathrm{xCT}$ is a cystine-glutamate exchange transporter that is upregulated in many cancers and is a biomarker for oxidative stress. Cationic AAs, including arginine, lysine, and histidine, are essential nutrients in proliferating cells. Arginine serves as a substrate for polyamine synthesis during cell proliferation and for nitric oxide synthesis. Additionally, therapies that deplete extracellular arginine are in clinical trials for cancers such as melanoma and hepatocellular carcinoma, and radiolabeled cationic AA transport substrates could serve as biomarkers for predicting and monitoring response to therapy.

\section{CONCLUSIONS AND FUTURE DIRECTIONS}

A range of radiolabeled AAs has been developed for tumor imaging. System L substrates such as ${ }^{11} \mathrm{C}$-MET and ${ }^{18} \mathrm{~F}$-FET are well established for imaging brain tumors and provide better estimates of gross tumor volumes and margins, better visualization of nonenhancing gliomas, and potentially earlier and more accurate assessment of response to therapy than is possible with MR imaging alone. Other important imaging applications with radiolabeled AAs include neuroendocrine tumor imaging with ${ }^{18} \mathrm{~F}$-FDOPA and prostate cancer imaging with ${ }^{18} \mathrm{~F}-\mathrm{FACBC}$. Tracers targeting other AA transport systems, including system A, glutamine, xCT, and cationic AA transporters, are under development, but their clinical utility remains to be established. Optimal application of this class of tracers for oncologic imaging will require matching the transport and metabolic properties of a given radiolabeled AA with the relevant tumor biology and clinical question.

\section{DISCLOSURE}

No potential conflict of interest relevant to this article was reported.

\section{REFERENCES}

1. Ganapathy V, Thangaraju M, Prasad PD. Nutrient transporters in cancer: relevance to Warburg hypothesis and beyond. Pharmacol Ther. 2009;121:29-40.

2. Nakanishi T, Tamai I. Solute carrier transporters as targets for drug delivery and pharmacological intervention for chemotherapy. J Pharm Sci. 2011;100:3731-3750.

3. Jewell JL, Guan KL. Nutrient signaling to mTOR and cell growth. Trends Biochem Sci. 2013;38:233-242.

4. Nicklin P, Bergman P, Zhang B, et al. Bidirectional transport of amino acids regulates mTOR and autophagy. Cell. 2009;136:521-534.

5. McConathy J, Yu W, Jarkas N, Seo W, Schuster DM, Goodman MM. Radiohalogenated nonnatural amino acids as PET and SPECT tumor imaging agents. Med Res Rev. 2012;32:868-905.

6. Singhal T, Narayanan TK, Jain V, Mukherjee J, Mantil J. $\left[{ }^{11} \mathrm{C}\right]-\mathrm{L}-$ methionine positron emission tomography in the clinical management of cerebral gliomas. Mol Imaging Biol. 2008;10:1-18.

7. Dunet V, Rossier C, Buck A, Stupp R, Prior JO. Performance of ${ }^{18}$ F-fluoro-ethyltyrosine $\left({ }^{18} \mathrm{~F}\right.$-FET) PET for the differential diagnosis of primary brain tumor: a systematic review and metaanalysis. J Nucl Med. 2012;53:207-214.
8. Fueger BJ, Czernin J, Cloughesy T, et al. Correlation of 6- ${ }^{18}$ F-fluoro-L-dopa PET uptake with proliferation and tumor grade in newly diagnosed and recurrent gliomas. J Nucl Med. 2010;51:1532-1538.

9. Wyss M, Hofer S, Bruehlmeier M, et al. Early metabolic responses in temozolomide treated low-grade glioma patients. J Neurooncol. 2009;95:87-93.

10. Pöpperl G, Kreth FW, Mehrkens JH, et al. FET PET for the evaluation of untreated gliomas: correlation of FET uptake and uptake kinetics with tumour grading. Eur J Nucl Med Mol Imaging. 2007;34:1933-1942.

11. Galldiks N, Stoffels G, Filss CP, et al. Role of $O-\left(2-{ }^{18} \mathrm{~F}\right.$-fluoroethyl $)$-L-tyrosine PET for differentiation of local recurrent brain metastasis from radiation necrosis. J Nucl Med. 2012;53:1367-1374.

12. Terakawa $\mathrm{Y}$, Tsuyuguchi $\mathrm{N}$, Iwai $\mathrm{Y}$, et al. Diagnostic accuracy of ${ }^{11} \mathrm{C}$-methionine PET for differentiation of recurrent brain tumors from radiation necrosis after radiotherapy. J Nucl Med. 2008;49:694-699.

13. Jager PL, Chirakal R, Marriott CJ, Brouwers AH, Koopmans KP, Gulenchyn KY. 6-L- ${ }^{18} \mathrm{~F}$-fluorodihydroxyphenylalanine PET in neuroendocrine tumors: basic aspects and emerging clinical applications. J Nucl Med. 2008;49:573-586.

14. Piccardo A, Lopci E, Conte M, et al. Comparison of ${ }^{18} \mathrm{~F}$-dopa PET/CT and ${ }^{123} \mathrm{I}-$ MIBG scintigraphy in stage 3 and 4 neuroblastoma: a pilot study. Eur $\mathrm{J} \mathrm{Nucl}$ Med Mol Imaging. 2012;39:57-71.

15. Schiesser M, Veit-Haibach P, Muller MK, et al. Value of combined 6- $\left[{ }^{18} \mathrm{~F}\right]$ fluorodihydroxyphenylalanine PET/CT for imaging of neuroendocrine tumours. Br J Surg. 2010;97:691-697.

16. Haug A, Auernhammer CJ, Wangler B, et al. Intraindividual comparison of ${ }^{68} \mathrm{Ga}-$ DOTA-TATE and ${ }^{18} \mathrm{~F}$-DOPA PET in patients with well-differentiated metastatic neuroendocrine tumours. Eur J Nucl Med Mol Imaging. 2009;36:765-770.

17. Schuster DM, Savir-Baruch B, Nieh PT, et al. Detection of recurrent prostate carcinoma with anti-1-amino-3- ${ }^{18} \mathrm{~F}$-fluorocyclobutane-1-carboxylic acid PET/ CT and ${ }^{111}$ In-capromab pendetide SPECT/CT. Radiology. 2011;259:852-861.

18. Oka S, Okudaira H, Yoshida Y, Schuster DM, Goodman MM, Shirakami Y. Transport mechanisms of trans-1-amino-3-fluoro $\left[1-{ }^{14} \mathrm{C}\right]$ cyclobutanecarboxylic acid in prostate cancer cells. Nucl Med Biol. 2012;39:109-119.

19. Kaira K, Oriuchi N, Shimizu K, et al. ${ }^{18} \mathrm{~F}-\mathrm{FMT}$ uptake seen within primary cancer on PET helps predict outcome of non-small cell lung cancer. $J$ Nucl Med. 2009;50:1770-1776.

20. Yu W, McConathy J, Williams L, et al. Synthesis, radiolabeling, and biological evaluation of $(R)$ - and $(S)$-2-amino-3-[ $\left.{ }^{18} \mathrm{~F}\right]$ fluoro-2-methylpropanoic acid (FAMP) and $(R)$ - and $(S)-3-\left[{ }^{18} \mathrm{~F}\right]$ fluoro-2-methyl-2- $N$-(methylamino)propanoic acid (NMeFAMP) as potential PET radioligands for imaging brain tumors. J Med Chem. 2010;53: $876-886$.

21. Lewerenz J, Hewett SJ, Huang Y, et al. The cystine/glutamate antiporter system $x$ (c) $(-)$ in health and disease: from molecular mechanisms to novel therapeutic opportunities. Antioxid Redox Signal. 2013;18:522-555.

22. Baek S, Choi CM, Ahn SH, et al. Exploratory clinical trial of $(4 S)-4-\left(3-\left[{ }^{18} \mathrm{~F}\right]\right.$ fluoropropyl)-L-glutamate for imaging $\mathrm{xC}-$ transporter using positron emission tomography in patients with non-small cell lung or breast cancer. Clin Cancer Res. 2012;18:5427-5437.

23. Qu W, Oya S, Lieberman BP, et al. Preparation and characterization of L[5- $\left.{ }^{11} \mathrm{C}\right]$-glutamine for metabolic imaging of tumors. J Nucl Med. 2012;53:98-105.

24. Qu W, Zha Z, Ploessl K, et al. Synthesis of optically pure 4-fluoro-glutamines as potential metabolic imaging agents for tumors. J Am Chem Soc. 2011;133: 1122-1133.

25. Turkman N, Gelovani JG, Alauddin MM. Radiosynthesis of $N 5-\left[{ }^{18} \mathrm{~F}\right]$ fluoroacetylornithine (N5-[ $\left.\left.{ }^{18} \mathrm{~F}\right] \mathrm{FAO}\right)$ for PET imaging of ornithine decarboxylase (ODC) in malignant tumors. J Labelled Comp Radiopharm. 2011;54:33-37.

26. McConathy J, Zhou D, Shockley SE, et al. Click synthesis and biologic evaluation of $(R)$ - and $(S)$-2-amino-3-[1-(2-[ $\left.{ }^{18} \mathrm{~F}\right]$ fluoroethyl)-1H-[1,2,3]triazol-4-yl]propanoic acid for brain tumor imaging with positron emission tomography. Mol Imaging. 2010;9:329-342. 